\title{
Application of Nano PAC on Mitigating Membrane Fouling by Surface Properties Optimization
}

\author{
MI Hairong ${ }^{\mathrm{a}}$, CHENG Gong ${ }^{\mathrm{b}}$ and DING Xuejiao ${ }^{\mathrm{c}}$ \\ College of Aerospace and Civil Engineering, Harbin Engineering University, Harbin 150001, China ${ }^{1}$ \\ a18045185613@163.com², b1943461464@qq.com, ${ }^{\mathrm{c}} 1321718240 @ q q . c o m$.
}

\begin{abstract}
Membrane material has been widely accepted owing to the ability in water treatment, but the membrane fouling problem in using process is seriously restricted the promotion of membrane technology. So in this research nano PAC flocculant is added into the raw water to reach the goals of strengthening treatment efficiency and mitigating membrane fouling. According to the TMP change, it results that the PAC dosage of $45 \mathrm{mg} / \mathrm{L}$ can realize the purpose to mitigate membrane fouling best. Compared with MBR, the MCBR is 16 days tardiness at the change of TMP, $1 / 3$ gel layer filtration resistance less and $3.5 \% \mathrm{C}$ element content fewer. The sum of $\mathrm{C}, \mathrm{O}$ and $\mathrm{N}$ elements content, in addition, in the gel layer pollutants is more than $95 \%$. That means membrane pollution is mainly caused by organic matter and nano PAC has the effect on surface properties optimization and membrane fouling mitigating.
\end{abstract}

\section{Introduction}

With the development of science and technology, the membrane which is a separable material with selectivity of material, is used to separate substance. Membrane technology is mainly composed of microfiltration, ultrafiltration, sodium filter, reverse osmosis in memory electric desalination ${ }^{[1]}$. The membrane is generally made of porous membrane so that acid and alkali will be got rid of in the process of water treatment of the traditional technology, and it can improve the water quality effectively ${ }^{[2]}$. Because of its efficient organic matter removal ability in the past few decades, membrane bioreactor (MBR) is considered to as the most promising wastewater treatment and recycling process. However, membrane fouling is a key problems which hinder the MBR widely used. It will increase the operating cost and the service life of the membrane will be shorten ${ }^{[3]}$.

Membrane fouling of MBR is generally divided into three pollution stages, when the filtration flux is constant, in every stage of pollution, the main influence factors is different ${ }^{[4]}$. The three stages of membrane fouling of in MBR are shown in figure 1. From the figure we can know membrane fouling is formed by the membrane pores, the membrane surface, and the microorganisms in sludge layer, so the membrane pollution has been a more concerned problem.

To solve the problem mentioned above, the researchers are attempting to find out an effective and economic way in all directions. For example, the membrane modification,

The Fundamental Research Funds for the Central Universities(HEUCFX1504)

* Corresponding author: 18045185613@163.com 
adding magnetic field or electric field, adding the adsorbent, optimizing experiment conditions and backwash conditions, etc ${ }^{[5]}$. The membrane modification is modified on the membrane surface or the structure of the membrane material reactive functional groups by some physical and chemical methods. Although this approach can mitigate the membrane fouling, but it can change the properties greatly and weaken the advantages of membrane material ${ }^{[6]}$. Optimal operation condition in the actual engineering does not need to change the reaction tank and equipment but more strict on operating conditions, but need to debug so many times ${ }^{[7]}$. Adding magnetic field, electric field, and adding the adsorbent are techniques which can mitigate the adhesion of pollutants and microorganism on the membrane surface, then mitigate the membrane fouling mainly through magnetic field, electric field and adsorbent for pollutants and the role of microorganisms in water. The technique of control operating condition is strict with practical engineering and increase the backwash, which is not economic ${ }^{[8]}$.

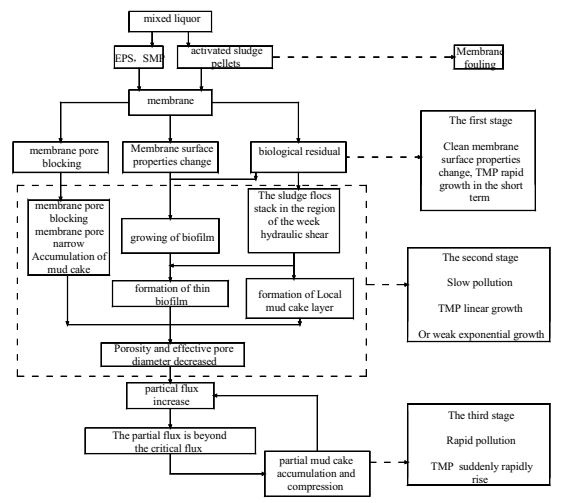

Fig. 1 The mechanism of membrane fouling of three stages in MBR

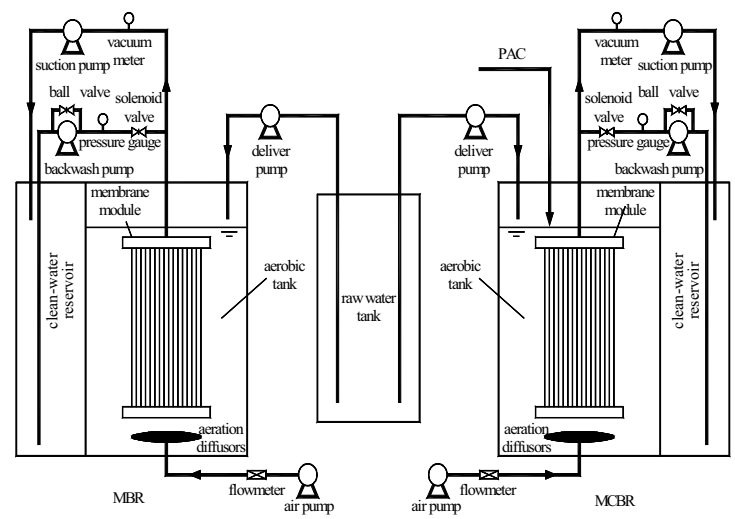

Fig. 2 The schematic diagram of experimental facility

Compared various process synthetically, and analyzed the mechanism of membrane fouling, nano PAC is added into MBR reactor to control the PAC dosage in this research. Its efficiency of mitigating the membrane fouling is analyzed through transmembrane pressure (TMP), Energy Disperse Spectroscopy (EPS), membrane fouling resistance distribution. 


\section{Experimental Materials and Methods}

Materials This experiment adopts two sets of identical MBR, the schematic diagram of experimental facility is shown in figure 2 . The reactor is made of organic glass, and the aerobic pool (reaction pool) which with two curtain membrane modules is $350 \mathrm{~mm}$ long, $400 \mathrm{~mm}$ wide, $500 \mathrm{~mm}$ high, and 46 liters effective volumes. The membrane module which made of polypropylene (PP) has $2 \mathrm{~m}^{2}$ effective areas and the pore size of the membrane is $0.1-0.2 \mathrm{um}$.

Operating Conditions The operation mode of the two reactors is continuous pumping without backwash and filtrating by constant flux of $1.2 \mathrm{~L} /\left(\mathrm{m}^{2} \cdot \mathrm{h}\right)$, dispose sewage of $4.8 \mathrm{~L} / \mathrm{h}$, hydraulic retention time of $9 \mathrm{~h}$. The reactors are continuously aerated by electromagnetic air pump with the aerated rate of $4 \mathrm{~m}^{3} / \mathrm{h}$. There is not sludge wastage except the reduce of some sludge when we are sampling the mixed liquor and cleaning the membrane, about 40d sludge detention time. The only difference of the two reactors is the MCBR have a coagulant adding system. We control the reactor running time through the TMP changes in the experimental. Ending the reactor operation when the TMP reached $50 \mathrm{kpa}$. The sludge comes from the returned sludge of the secondary sedimentation tank at Harbin Xinyi municipal sewage treatment plant. This test is proposed to deal with municipal wastewater, simulate the municipal wastewater by using chemicals according to the content of the main pollutants in municipal wastewater ${ }^{[9]}$.

\section{Experimental Methods}

Optimal Dosage of PAC It is experimented that different PAC concentration from low to high were added into the reactor (we translate the PAC concentration into Al concentration at here and we use the $\mathrm{Al}$ concentration expressing the PAC concentration at all the paper) to text the operation condition of the MBR under different concentrations of Al. We pay $6 \mathrm{~h}$ for each coagulation experiment, take water sample every $30 \mathrm{~min}$ examine the change of TMP every one hour under different concentrations of Al.

Change of TMP On the condition of constant flux, the increased rate of TMP is an important indicator for the property of MBR, because it can directly reflect the degree of membrane pollution from the macroscopic. We use the vacuum meter reading represent the TMP value, and read once a day in the course of the experiment. When the TMP reached $50 \mathrm{kpa}$ ends the experiment, and begins backwash.

Pollution Layer Resistance According to the filtration resistance model, the relationship between membrane flux and the TMP can be represented by the following equation: 


$$
\begin{array}{r}
J=\frac{\Delta P}{\mu R_{\mathrm{t}}} \\
(1) \\
R_{\mathrm{t}}=R_{m}+R_{c}+R_{g}+R_{i}
\end{array}
$$

In the equation we use the ' $\mathrm{J}$ ' to denote membrane flux, the ' $\Delta \mathrm{P}$ ' denote TMP, the ' $\mu$ ' is for dynamic viscosity of liquid filtration, the ' $\mathrm{R}_{\mathrm{t}}$ ' to represent the total filtration resistance in membrane, the ' $R_{m}$ ' is for membrane autologous filtration resistance, the ' $R_{c}$ ' is for the filtration resistance of cake layer formed by activated sludge, the ' $\mathrm{R}_{\mathrm{g}}$ ' to denote the filtration resistance of gel layer formed by biopolymer, and the ' $R_{i}$ ' to represent the filtration resistance of inorganic pollutant. The membrane surface is washed with water to remove the cake layer, then filter the membrane with pure water. So we can figure out $R_{\mathrm{m}}+R_{\mathrm{g}}+R_{\mathrm{i}}$ by this membrane flux and TMP. After that we wash the membrane surface by alkaline liquor to remove the gel layer, then filter the membrane with pure water. So we can figure out $R_{\mathrm{m}}+R_{\mathrm{i}}$ by this membrane flux and TMP. Finally, we wash the membrane surface by acid liquor to remove the inorganic pollutant, then filter the membrane with pure water. So we can figure out $R_{\mathrm{m}}$ by this membrane flux and TMP.

Energy Dispersive Spectroscopy(EDS) In this experiment, we use X-ray spectrometer of scanning electron microscopy (S-4800, HITACHI, JAPAN) to analyze the elementary composition of the pollutant layer $(1-3 \mu \mathrm{m})$ in the membrane surface. We analysis the material chemical composition of the sample, according to the characteristic spectral lines formed at the transition process of different element shell electrons. And then we can carry out the qualitative and semi-quantitative analysis of macro element.

\section{Experimental Results}

Dosage of PAC The change of TMP within MBR run for $6 \mathrm{~h}$, when adding the different concentrations of $\mathrm{Al}$, was shown in figure 3 .

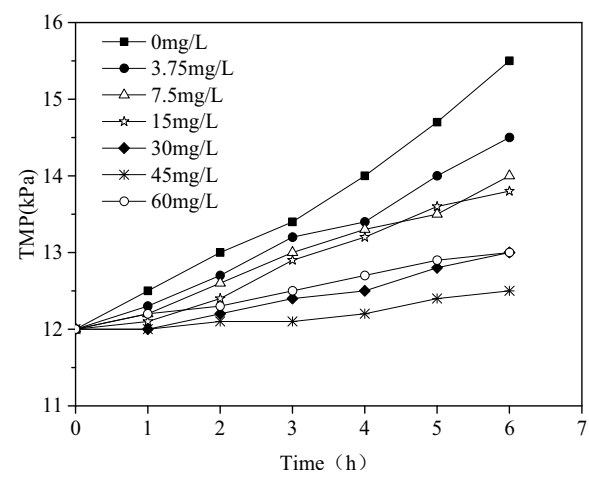

Fig. 3 The change of TMP under different concentrations of $\mathrm{Al}$ 


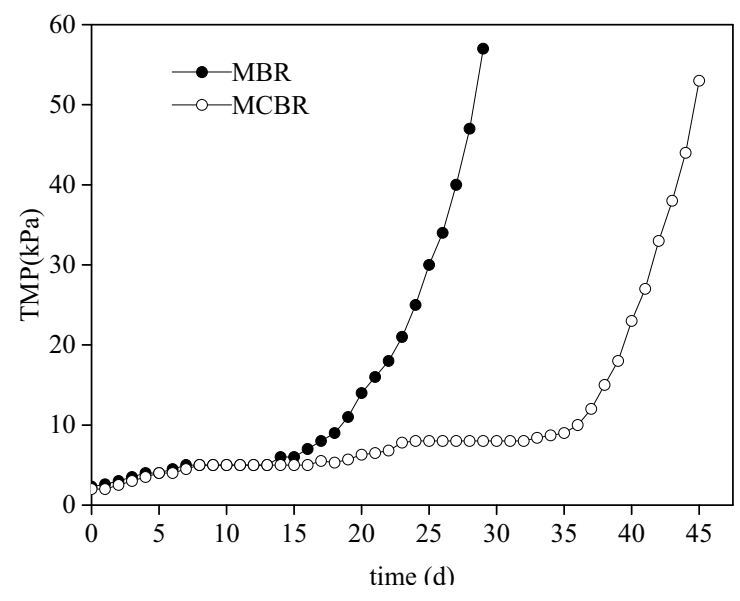

Fig. 4 The TMP change of MBR and MCBR with time

The figure 3 shows that, when adding Al concentration by 3.75, 7.5, 15, 30, 45 and 60 $\mathrm{mg} / \mathrm{L}$, all concentrations can effectively slow the growth of TMP. But at the Al concentration of $45 \mathrm{mg} / \mathrm{L}$, the growth rate of TMP is the most slowly, and it can get the best results to mitigate the membrane fouling.

Change of TMP with Time The TMP of MBR and MCBR changed with the reactor running every day, and the result were shown in figure 4.

It is known from the figure 4 that the TMP of MBR reached to 50kpa only in 29 days, but TMP of MCBR didn't reach to 50kpa until 45 days. When the TMP were up to 50kpa, MCBR run for 16 days more than MBR, it can directly indicate that adding PAC could effectively mitigate the membrane fouling rate of membrane bioreactor.

Pollution Layer Resistance After the experiment of TMP, we calculate the pollution layer resistance according to the methods of 2.3.3, then we can get the resistance like table 1.

TABLE 1 DISTRIBUTION OF POLLUTION LAYER RESISTANCE

\begin{tabular}{|c|c|c|c|c|c|c|}
\hline \multirow{2}{*}{ Reactor } & \multicolumn{2}{|c|}{$R_{\mathrm{c}}$} & \multicolumn{2}{c|}{$R_{\mathrm{g}}$} & \multicolumn{2}{c|}{$R_{\mathrm{i}}$} \\
\cline { 2 - 7 } & $10^{11}\left(\mathrm{~m}^{-1}\right)$ & $\%$ & $10^{11}\left(\mathrm{~m}^{-1}\right)$ & $\%$ & $10^{11}\left(\mathrm{~m}^{-1}\right)$ & $\%$ \\
\hline MBR & 1.89 & 67.4 & 9.11 & 32.5 & 3.14 & $<0.1$ \\
\hline MCBR & 5.3 & 91 & 5.23 & 9 & 4.73 & $<0.1$ \\
\hline
\end{tabular}

From table 1 we can know that the ' $\mathrm{R}_{\mathrm{c}}$ ' has greatest contribution to the total resistance $\mathrm{R}_{\mathrm{t}}$, and accounts for the percentage of the total resistance is $67.4 \%-91 \%$. It is shown, comparing the filtration resistance of the two reactor (MBR, MCBR) gel layer, that the gel layer filtration resistance in the MCBR is less than $1 / 3$ of MBR, even thought the filtering time of the MCBR is nearly 1.5 times of MBR. By the calculation, the gel layer filtration resistance average growth rate of MCBR and MBR were $1.16 \times 10^{9} \mathrm{~m}^{-1} / \mathrm{d}$ and $3.14 \times 10^{9} \mathrm{~m}^{-1} / \mathrm{d}$, the average growth rate of the MCBR is nearly three times slower than MBR. That is to say the cumulative rate of inorganic pollutants in the MCBR is slower than MBR, and the PAC will not lead to more serious inorganic pollution.

Energy Dispersive Spectroscopy (EDS) Analysis In this experiment, it can be known, by using the experimental apparatus shown at 2.3.4, that the elementary composition of the 
gal layer pollution in MBR and MCBR. And the result is shown at table 2.

TABLE 2 POLLUTANT EDS ANALYSIS

\begin{tabular}{|c|c|c|c|}
\hline \multirow{2}{*}{ Elements } & \multicolumn{2}{|l|}{ MBR } & \multirow{2}{*}{$\begin{array}{c}\text { MCBR } \\
\text { Weight Percentage }(\%)\end{array}$} \\
\hline & Weight Percentage & $(\%)$ & \\
\hline $\mathrm{C}$ & 49.65 & & 46.15 \\
\hline $\mathrm{O}$ & 32.99 & & 33.23 \\
\hline $\mathrm{N}$ & 14.33 & & 17.13 \\
\hline $\mathrm{Al}$ & 1.23 & & 1.18 \\
\hline $\mathrm{Cl}$ & 0.43 & & 0.84 \\
\hline $\mathrm{Fe}$ & 0.40 & & 0.56 \\
\hline $\mathrm{Ca}$ & 0.38 & & 0.43 \\
\hline $\mathrm{Na}$ & 0.36 & & 0.29 \\
\hline $\mathrm{Mg}$ & 0.17 & & 0.19 \\
\hline
\end{tabular}

It can be found from table 2 that there are a large number of $\mathrm{C}$ and $\mathrm{O}$ elements which represent organic pollutants and a small amount of inorganic elements such as metallic elements $\mathrm{Al}, \mathrm{Fe}, \mathrm{Ca}, \mathrm{Na}$, etc. This shows that the membrane fouling of membrane bioreactor is formed by organic and inorganic pollution. The table 2 also shows that the sum of $\mathrm{C}, \mathrm{O}, \mathrm{N}$ elements content in the gel layer pollutants of the MBR and MCBR is more than $95 \%$. This shows that the organic pollution of membrane pollution is much bigger valuable than inorganic pollution, and membrane pollution In the MBR and MCBR is mainly caused by organic pollution. Further, the $\mathrm{C}$ element content in the gel layer of MCBR was lower than MBR, This shows that the gel layer organic pollution in MBR is more serious than MCBR, also explains that adding PAC can improve the gel layer pollutants of membrane surface to some extent, and mitigate the organic pollution.

\section{Conclusions}

Compared the effect on TMP with adding different concentration of PAC on the short-term experiments, ultimately determine the optimal dosage of PAC to $45 \mathrm{mg} / \mathrm{L}$. This concentration is as the PAC dosage for a long-term experiment of MCBR running. Long-term experiments with nano PAC can effectively mitigate the membrane fouling. Through the TMP, it is shown that MCBR run for 16 days more than MBR to 50kpa, which means nano PAC can resist membrane fouling effectively. Analyzing the membrane resistance, the percentage of ' $R_{c}$ ' in total resistance can reach $67.4 \%-91 \%$, and the gel layer filtration resistance in the MCBR is $2 / 3$ of that in the MBR. We can get the results that nano PAC can resist membrane fouling by control the gel layer filtration resistance mainly. According to the energy dispersive spectroscopy analysis (EDS), it is proved that the sum of $\mathrm{C}, \mathrm{O}, \mathrm{N}$ elements content, both at MBR and MCBR, of the gel layer pollutants is more than $95 \%$. Besides, the $\mathrm{C}$ element in MCBR is 3.5\% less than MBR, which represents the organic pollution has a larger contribution to membrane fouling than the inorganic pollution, and the nano PAC will not lead to a more serious inorganic pollution. As a consequence, dosing nano PAC could be an effective method in membrane fouling mitigating by surface properties optimization. 


\section{References}

1. Yang, Q., Chen, J., Zhang, F. Membrane fouling control in a submerged membrane bioreactor with porous, flexible suspended carriers[J]. Desalination, 2006, 189(1-3):292-302.

2. Gomez, M., Dvorak, L., Ruzickova, I., Holba, M., Wanner, J. Operational experience with seasonally operated full-scale membrane bioreactor plant $[\mathrm{J}]$. Bioresour. Technol., 2012, 121:241-247

3. Dvorak, L., Gomez, M., Dvorakova, M., Ruzickova, I., Wanner, J. The impact of different operating conditions on membrane fouling and EPS production[J]. Bioresour. Technol., 2011, 102(13):6870-6875

4. Holba, M., Ploteny, K., Dvorak, L., Gomez, M., Ruzickova, I. Full-scale application of membrane filtration in municipal wastewater treatment plants[J] CSAW, 2012, 40(5):479-486

5. Dizge, N., Koseoglu-Imer, K.Y., Karagunduz, A., Keskinler, B. Effects of cationic polyelectrolyte on filterability and fouling reduction of submerged membrane bioreactor(MBR)[J]. J. Membr. Sci., 2011, 377(1-2):175-181

6. Ngo, H.H., Guo, W. Membrane fouling control and enhanced phosphorus removal in and aerated submerged membrane bioreactor using modified green bioflocculant $[\mathrm{J}]$. Bioresour. Technol., 2009, 100(18):4289-4291

7. G.T. Seo, C.D. Moon, S.W. Chang, S.H. Lee, Long term operation of high concentration powdered activated carbon membrane bio-reactor for advanced water treatment[J]. Water Sci. Technol., 2004, 50(8):81-87

8. Yang, X.L., Song, H.L., Lu, J.L., Fu, D.F., Cheng, B. Influence of diatomite addition on membrane fouling and performance in a submerged membrane bioreactor[J]. Bioresour. Technol., 2010, 101(23):9178-9184

9. P.Le-Clech, V.Chen, T.A.G.Fane. Fouling in membrane bioreactors used in wastewater treatment[J]. J. Membr. Sci., 2006, 284(1-2): 17-53. 